\title{
Interfacial interaction driven rheological properties of quartz nanofluids from molecular dynamics simulations and density functional theory calculations
}

\section{Zhaoyang Lou ( $\sim$ louslove@163.com )}

Affiliated Cancer Hospital of Zhengzhou University https://orcid.org/0000-0002-1336-7261

Chen Cheng

Affiliated Cancer Hospital of Zhengzhou University

Yingqi Cui

Sichuan University Institute of Atomic and Molecular Physics

Hao Tian

Sichuan University Institute of Atomic and Molecular Physics

\section{Research Article}

Keywords: Quartz nanofluids, Viscosity, Molecular dynamics simulation, Density functional theory calculation

Posted Date: January 17th, 2022

DOI: https://doi.org/10.21203/rs.3.rs-1235353/v1

License: (c) (1) This work is licensed under a Creative Commons Attribution 4.0 International License. Read Full License 


\section{Abstract}

Correlations of the shear viscosity of quartz nanofluids with particle concentration, particle size, and temperature were investigated with molecular dynamics simulations and density functional theory (DFT) calculations. A new understanding to the experimentally concluded correlations was addressed in terms of microscopic particle-water interfacial interaction in three aspects. First, the viscosity of quartz nanofluids at different particle concentrations, particle sizes and temperatures was simulated using the equilibrium molecular dynamics simulations method to reproduce the experimental observations. The important role of particle-water interaction in the viscosity determination of nanofluids was revealed. Second, DFT calculations confirm a stronger particle-water interaction than that among water molecules. Finally, a correlation was proposed to fit the simulated results and compared with earlier twoparameter correlations. One parameter in the correlation is indeed a constant, while the other is a function of $\mathrm{SiO}_{2}$-water interaction energy. Our study proposes a physical basis for the experimentally concluded correlations on the viscosity of nanofluids.

\section{Introduction}

Nanofluids are usually engineered by dispersing nano-sized particles in a base fluid such as water, ethylene glycol, propylene glycol and so on. In the past decades nanofluids were broadly used in many industries including power generation, chemical processes, heating and cooling processes, transportation, microelectronics and other micro-sized applications because of their enhanced thermal and mechanical properties [1-5]. In fact, most of these applications make use of the unique rheological properties of nanofluids that vary with particle type, size, shape and amount, in addition to the intrinsic properties of base fluids. Knowledge of the rheological behavior of nanofluid is therefore crucial in shaping their practical applications.

Many studies have been carried out on the rheological properties of nanofluids [6-14]. Minakov [6] systematacially measured the viscosity of more than 30 different nanofluids based on distilled water, ethylene glycol and engine oil, suggesting that their viscosity increases with decreasing particle diameter. Ezekwem [7] proposed a relationship of viscosity with temperature and volume fraction for AIN and SiC nanofluids. The nanofluid viscosity decreases significantly with temperature, and increases with

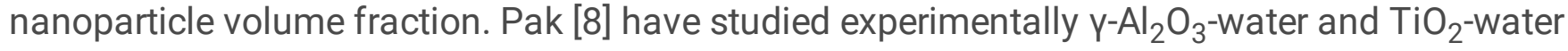
nanofluids and found their viscosity increases with increasing particle concentration. Moreover, the viscosity magnitudes are significantly greater than those from Batchelor equation [9]. Wang [10] reported a maximum enhancement of $86 \%$ for the viscosity of $\mathrm{Al}_{2} \mathrm{O}_{3}(28 \mathrm{~nm})$-water nanofluids. Similar viscosity increments were also observed by Wole-Osho [15]. In a study for CuO nanofluids, Kulkarni [12] correlated the viscosity, temperature and particle concentration in the form of , where $\mu_{\mathrm{s}}$ is the suspension viscosity and $A$ and $B$ are two parameters related to volume concentration.

Even though these studies have revealed the rheological dependence of some nanofluids from macroscopic measurements, little is known about the molecular mechanism of how particle size and 
concentration affect the rheological properties of nanofluids. While the particle size and shape distributions are difficult to control in experiments, computer simulations provide a useful approach to explore such correlations explicitly. As molecular dynamics (MD) methods have been proven to be an effective and reliable approach to investigate the microscopic structures, rheological and related properties of various gases, liquids, and solids [16-20], we carried out equilibrium molecular dynamics simulations and density functional theory (DFT) calculations on the quartz-in-water nanofluids in this work, aiming to reveal the origin of their viscosity dependence on particle size, particle concentration and temperature. Quartz, abundant on the earth and easy in pulverization, is commonly used in the preparation of nano-additives. The addition of quartz nanoparticles leads to various improvements in rubber, plastic and coating products. In most processes, quartz nanoparticles are dispersed into water or other fluids for ease of usage. Understanding to the rheological behavior of quartz nanofluids from their molecular mechanism could be helpful with their applications in manufacture and for the development of other kinds of nanofluids.

\section{Materials And Simulation Methods}

Two approaches, equilibrium and nonequilibrium, were often used to calculate the viscosity in MD simulations [17, 20-23]. The nonequilibrium approach had ever been considered more efficient from a computational point of view than the equilibrium one that sometimes suffers from poorly converged viscosity. However, Chen [23] and Guo [24] have clarified that the convergence issues can be addressed with enough statistics and by a careful selection of the integration times. The equilibrium approach does not suffer from additional adjustments that the nonequilibrium approach usually needs. In addition, the equilibrium MD is a multi-property method with which all thermodynamic properties can be computed at the same state point from a single simulation run. Using an equilibrium simulation method Wang [25] have studied the mechanism of heat flow in a model nanofluid system. The equilibrium MD simulations were therefore employed in this work. The viscosity is calculated by the Green-Kubo integral formula [26, 27],

$$
\mu_{s}=\frac{V}{k_{B} T} \int_{0}^{\infty}\left\langle P_{\alpha \beta}(t) P_{\alpha \beta}(0)\right\rangle d t
$$

in the equilibrium approach. $\mu_{s}$ is the shear viscosity, $V$ is the volume of the system, $T$ is the temperature, $k_{B}$ is the Boltzmann constant, and $P_{a \beta}$ are off-diagonal components of the pressure tensor.

A periodic $30 \times 30 \times 30 \AA^{3}$ cell was built to mimic the quartz-water system. The quartz particles were cut in the shape of a cube from bulk a-quartz. The Si and $\mathrm{O}$ atoms were arranged alternatively on the particle surface. The quartz particles were then randomly placed in the cell and the rest space was homogeneously filled with water molecules. The water density was set to $1.0 \mathrm{~g} / \mathrm{mL}$ for all the starting structures. The diameter (the diagonal length of the cube) and number of quartz particles can be tuned to model the nanofluids with different concentrations. A snapshot of the $\mathrm{SiO}_{2}-$ water cell was shown in $\mathrm{Fig}$ 1. In the MD simulation, the TIP4P/2005 force field for water and the CLAYFF force filed for $\mathrm{SiO}_{2}$ were 
used. The former has been widely used in the simulations for water-containing systems [30-32] and the latter was developed for clay systems and has shown great feasibility for water/clay systems. A typical simulation was carried out in three successive steps with the LAMMPS package [36]. First, the system was subjected to an NPT dynamics for 800 ps using a time step of $1 \mathrm{fs}$ at the temperature of interest. In this step the cell volume was adjusted to match the density of the system at the target temperature. The system was then equilibrated for more than 100 ps in an NVT ensemble until its energy fluctuation become stable. Finally the production steps of $10 \mathrm{~ns}$ were performed.

The simulations were conducted over a temperature range of $280-340 \mathrm{~K}$, which was of interest in practical applications. A Nose-Hoover thermostat [37] was used to maintain the system temperature. Long range electrostatic interactions were computed using the Particle-Particle Particle-Mesh K-space technique and a cut-off of $10 \AA$ was used for short-range interactions. The components of pressure tensor were accumulated at every time step. The pressure autocorrelation functions (ACFs) and the shear viscosity coefficient were then obtained by numerically integrating the components over a time window of $5 \sim 10$ ps depending on the systems.

With these settings, we computed the shear viscosity of water, which is the solvent of $\mathrm{SiO}_{2}$ nanofluid, to validate the above computational strategy. The simulations were carried out over $280-340 \mathrm{~K}$ with an interval of $10 \mathrm{~K}$. The results, as shown in Table 1, are in good agreement with previous experiments [38] for both shear viscosity and density. The maximum deviations are less than $5 \%$.

The DFT calculations were carried out under the generalized gradient approximation (GGA) of PerdewBurke-Ernzerhof (PBE) parameterization [39], as implemented in the $\mathrm{DMol}^{3}$ package [40]. Both the cluster model and the slab model geometry optimization calculations were performed using the double numerical polarization (DNP) basis set [40] which considers a polarization $d$ function on heavy atoms and a polarization $p$ function on hydrogen atoms. A Grimme-type [41] dispersion potential was used to describe the interatomic weak interaction in the systems. Periodic boundary condition was applied to the slab model to mimic the interactions between water molecule and surface. The slabs were separated from their images in the neighboring cells by a vacuum width of $30 \AA$, a distance large enough to avoid the interactions between neighboring slabs. For all the geometry optimizations the convergence criteria were set to 0.004 a.u. on the gradient, 0.005 a.u. on the displacement and $2.0 \times 10^{-5}$ a.u. on the energy.

\section{Results And Discussion}

The viscosity of $\mathrm{SiO}_{2}$ nanofluid was simulated at constant temperatures for the systems with different volume concentrations, which are defined as the volume fraction of $\mathrm{SiO}_{2}$ particles. As shown in Fig $2 \mathrm{a}$, the viscosity increases with $\mathrm{SiO}_{2}$ concentration at a given temperature. Moreover, the increase is more significant at lower temperatures. When the concentration increases from $1.2 \%$ to $4.8 \%$, for example, the viscosity increases by $1.47 \mathrm{mPa} \cdot \mathrm{s}$ at $280 \mathrm{~K}$ and $0.32 \mathrm{mPa} \bullet \mathrm{s}$ at $340 \mathrm{~K}$, respectively. For these simulations, all the $\mathrm{SiO}_{2}$ particles are fixed at $11.2 \AA$ in diameter. A large volume concentration means more particles in the system. The increasing $\mathrm{SiO}_{2}$ particle number increases the particle-water contacting area, leading 
to increasing contribution from particle-water interaction to the viscosity. As we will show below, the particle-water interaction, which is stronger than water-water interaction, tends to increase the viscosity. The volume concentration dependence of nanofluids has been studied experimentally for quartz, copper oxide, titanium dioxide systems [4, 13, 42, 43]. In Namburu's measurements [42], the high concentrations of quartz nanoparticles in ethylene glycol and water mixture leads to great viscosity, and the viscosity variations at low temperature is more significant than that at high temperature. Our calculations produced similar results with the observations.

The temperature dependence of fluids has been well addressed by many authors [4, 11, 12, 42-44]. Increased molecular kinetic energy at high temperature usually makes the fluid viscosity small. This is true for $\mathrm{SiO}_{2}$ nanofluid. Fig $2 \mathrm{a}$ also shows the temperature dependence of viscosity for the nanofluid at a given $\mathrm{SiO}_{2}$ concentration. Similar to water, the viscosity of $\mathrm{SiO}_{2}$ nanofluid decreases with temperature. However, the temperature sensitivity of viscosity is different for the systems. The viscosity of systems with higher $\mathrm{SiO}_{2}$ concentration drops more rapidly with temperature. In Fig 2a, the slope of viscositytemperature curves increases with $\mathrm{SiO}_{2}$ concentration, indicating that the $\mathrm{SiO}_{2}$-water interaction is more important in viscosity contribution at lower temperature. This holds only when the interaction between $\mathrm{SiO}_{2}$-water interfaces is stronger than that between water molecules.

For $\mathrm{SiO}_{2}$ nanofluids with a given volume concentration, their viscosity depends on the particle size. In Fig 3 we compare the viscosity of $\mathrm{SiO}_{2}$ nanofluid with different particle diameters. The large particle, about 18.4 $\AA$ in diameter, is larger than the small one $(11.2 \AA$ ) by $170 \%$ in surface area and by $343 \%$ in volume. Since the total volume fraction of $\mathrm{SiO}_{2}$ particles is fixed, the system with smaller particle size has greater particle number. As a result, the total surface area increases by a ratio of 18.4:11.2. Therefore, $\mathrm{SiO}_{2}-$ water interface interaction plays a more significant role in the system with smaller particle size. Our calculations reveal that at every temperature the system with smaller particles has larger viscosity. Moreover, the difference becomes more remarkable at lower temperature. For example, the small $\mathrm{SiO}_{2}$ particles result in a viscosity of $0.78 \mathrm{mPa} \cdot \mathrm{s}$ at $340 \mathrm{~K}$, only $0.15 \mathrm{mPa} \cdot \mathrm{s}$ larger than that by the large particles. At $280 \mathrm{~K}$, however, their viscosity difference becomes $1.03 \mathrm{mPa} \bullet \mathrm{s}$. Namburu [42] measured the viscosity of $\mathrm{SiO}_{2}$ nanoparticles with various diameters of 20,50 and $100 \mathrm{~nm}$ suspended in a 60:40 (by weight) ethylene glycol and water mixture in a wide temperature range from -35 to $50^{\circ} \mathrm{C}$, revealing that at same volume fraction the nanofluids with large particle diameters have low viscosity. Our calculated results are consistent with the experimental observations for both the particle size dependence and its variation with temperature.

It is interesting to look into the relative viscosity, which was often used to measure the viscosity of nanofluids. The relative viscosity is defined as the viscosity ratio of nanofluid with respect to pure solvent, $\mu_{r}=\mu_{s} / \mu_{w}$ where $\mu_{w}$ is the viscosity of water. The computed $\mu_{r}$ values for $\mathrm{SiO}_{2}$ nanofluids are shown in Fig 2 b. Remarkable concentration dependence can be noted. The ratios are about 1.1, 1.3, 1.6, and 2.2 for the four concentrations, and nearly unchanged within the temperature range except that for the highest concentration of $4.8 \%$. Such terraced increase of $\mu_{r}$ with respect to concentration confirms that the 
viscosity increase mainly comes from $\mathrm{SiO}_{2}$-water interaction rather than water-water or $\mathrm{SiO}_{2}-\mathrm{SiO}_{2}$ interaction. It has been observed that the relative viscosity of copper oxide nanofluid has very small changes (less than 0.3 ) over -35 to $50^{\circ} \mathrm{C}$ at low concentrations [43]. The $\mu_{r}$ decay at high temperature for the samples with high concentrations was also noted. Similar results were also reported by Prasher for alumina particles suspended in propylene glycol with a volume concentration of $0.5 \%, 2 \%$, and $3 \%$ at $30-$ $50^{\circ} \mathrm{C}$ [11]. Our calculations reveal that the $\mu_{r}$ of $\mathrm{SiO}_{2}$ nanofluid is concentration dependent instead of temperature dependent at low $\mathrm{SiO}_{2}$ concentrations. In addition, we also noted the decay of $\mu_{r}$ at high temperature (above $300 \mathrm{~K}$ ) for systems with relatively high volume concentrations of $3.6 \%$ and $4.8 \%$.

Above calculations demonstrate the important role of $\mathrm{SiO}_{2}$-water interaction. It is therefore interesting to inspect such kind of interaction further. Two computational models were then designed to evaluate the interacting patterns between $\mathrm{SiO}_{2}$ and water by means of DFT calculations, as shown in Fig 4. One is the cluster model in which a water molecule adsorbs onto a $\left(\mathrm{SiO}_{2}\right)_{6}$ cluster. The structure of $\left(\mathrm{SiO}_{2}\right)_{6}$ cluster was taken from Ref. [[45]]. The water $\mathrm{H}$ atom binds with one of the $\mathrm{O}$ atoms of the cluster via a hydrogen bond. The interaction energy, which is defined as the energy difference between the systems before and after water adsorption, is about $1.43 \mathrm{eV}$. A similar cluster model, a water molecule adsorbing onto a $\left(\mathrm{H}_{2} \mathrm{O}\right)_{6}$ cluster, which was taken from Ref. [[46]], gives the interaction energy of $0.65 \mathrm{eV}$ between the water molecule and the water cluster. In the second model, periodical DFT calculations were performed to compute the interaction of a water molecule on the $\mathrm{SiO}_{2}(001)$ and ice (001) surfaces, which were sliced respectively from a-quartz and cube-ice crystal structures. Under this slab model, the computed interaction energy is $1.78 \mathrm{eV}$ for a water molecule on the $\mathrm{SiO}_{2}$ surface and $0.94 \mathrm{eV}$ on the ice surface. Larger interaction energies between $\mathrm{SiO}_{2}$ and water were predicted by both the cluster and the slab models, confirming above speculations from MD computations at the force-field level.

Several expressions have been proposed by Bicerano, [47] Brinkman, [48] Duangthongsuk, [4] Kulkarni [12] and Namburu [43] to fit the measured viscosity data of nanofluids, providing an estimation for viscosity variation with particle concentration and/or temperature. Most of these correlations are similar in nature, though different parameters were used to adjust the values for high concentration systems. The effect of particle size, however, is ignored in these correlations. As we found above, the nanofluid systems with different particle sizes may have quite different viscosities even though they have the same volume concentration. Our MD and DFT calculations revealed the decisive role of $\mathrm{SiO}_{2}$-water interaction in the rheological behavior of $\mathrm{SiO}_{2}$ nanofluids. We would explore below the correlation of the viscosity of $\mathrm{SiO}_{2}$ nanofluids with particle-water interaction strength.

Starting from the data in Fig 2, an exponential correlation,

$\mu_{\mathrm{s}}=\mathrm{A} \mathrm{e}^{-\mathrm{B} / \mathrm{T}}(2)$

can be fitted. A good correlation with $R^{2}>0.99$ is obtained, as shown in Fig 5. The systems with different $\mathrm{SiO}_{2}$ concentrations have similar slopes $(\ln A)$. The concentration effect on the viscosity is then 
represented only in parameter $B$. From (2) one has

$\ln \mu_{\mathrm{r}}=\mathrm{A}^{\prime}-\Delta \mathrm{B} / \mathrm{T}(3)$

where $A^{\prime}$ is a constant and $\Delta B=B-B_{w}$. $B_{w}$ is fitted from the viscosity of pure water. Eq. (3) has the similar form with the correlations proposed by Kulkarni [12] and Namburu [43] in which both $A$ and $B$ were functions of particle concentrations. From our MD simulations, the $\mathrm{SiO}_{2}$-water interaction energy $\left(E_{\text {int }}\right)$ can be obtained by summing up the coulomb and van der Waals terms between $\mathrm{SiO}_{2}$ particles and water molecules. Fig 6 presents the correlation of $\Delta B$ with $E_{\text {int }}$. It is interesting to note that with increasing $\mathrm{SiO}_{2}$ water interaction energy $\Delta B$ increases, leading to increasing $\mu_{r}$ Therefore, the concentration dependent parameters in previously observed correlations $[12,43]$ for nanofluids can be further understood as quantities relating to particle-solute interaction, and can be expressed as functions of interaction energy.

\section{Conclusion}

The rheological properties, in particular to the viscosity variations, of $\mathrm{SiO}_{2}$ nanofluids with particle concentration, particle size and temperature were simulated using the equilibrium molecular dynamics method and density functional theory calculations. Our calculations reproduce the experimental observations that the viscosity of $\mathrm{SiO}_{2}$ nanofluids increases with particle concentration and decrease with temperature. At a fixed volume faction, moreover, the viscosity increases with decreasing particle size. The viscosity variations were rationalized in terms of the particle-water and water-water interactions in the nanofluid systems. DFT calculations with both cluster and slab models reveal the interaction between $\mathrm{SiO}_{2}$ particle and water is much stronger than that between water molecules, which is responsible for the viscosity variation of $\mathrm{SiO}_{2}$ nanofluids. Furthermore, a correlation was proposed to fit the simulated results and compared with earlier correlations. A new understanding to the parameters in previously observed correlations was proposed from microscopic particle-water interfacial interaction. One parameter is a constant, while the other is a function of $\mathrm{SiO}_{2}$-water interaction energy.

\section{Declarations}

Funding: This work was funded by the Scientific Research Foundation for Doctoral of the Affiliated Cancer Hospital of Zhengzhou University.

Conflict of interest: The authors declare no competing interests.

Availability of data and material: All data generated or analyzed during this study are included in this published article.

Code availability: Not applicable.

Author Contributions: Zhaoyang Lou and Chen Cheng contributed equally to the study for data analyzing and writing. Yingqi Cui and Hao Tian performed the computational work and collected the data. All 
authors revised the draft manuscript and approved the final version.

Acknowledgements: We thank the High-Performance Computers for Physics of Sichuan University for providing us with computer time.

\section{References}

1. Amin AM, Hamzah WAW, Oumer AN (2021) Thermal conductivity and dynamic viscosity of mono and hybrid organic- and synthetic-based nanofluids: A critical review. Nanotechnol Rev 10:16241661

2. Jin C, Wu QB, Yang GQ, Zhang HY, Zhong YF (2021) Investigation on hybrid nanofluids based on carbon nanotubes filled with metal nanoparticles: Stability, thermal conductivity, and viscosity. Powder Technol 389:1-10

3. Zhang XB, Zhang HY, Lin J, Li LP, Wu QG (2014) Thermal conductivity and thermal stability enhancement of ethylene propylene diene methylene with carbon nanotube. J Reinf Plast Comp 33:767-774

4. Duangthongsuk W, Wongwises S (2009) Measurement of temperature-dependent thermal conductivity and viscosity of $\mathrm{TiO}_{2}$-water nanofluids. Exp Therm Fluid Sci 33:706-714

5. Choi SUS (2009) Nanofluids: From Vision to Reality Through Research. J Heat Trans-t Asme 131:033106

6. Minakov AV, Rudyak VY, Pryazhnikov MI (2021) Systematic Experimental Study of the Viscosity of Nanofluids. Heat Transf Eng 42:1024-1040

7. Ezekwem C, Dare A (2020) Experimental investigation on viscosity of AIN and SiC nanofluids. Chem. Eng. Commun.:17

8. Pak BC, Cho YI (1998) Hydrodynamic and heat transfer study of dispersed fluids with submicron metallic oxide particles. Exp Heat Transfer 11:151-170

9. Batchelor GK (1977) The effect of Brownian motion on the bulk stress in a suspension of spherical particles. J Fluid Mech 83:97-117

10. Wang XW, Xu XF, Choi SUS (1999) Thermal conductivity of nanoparticle-fluid mixture. J Thermophys Heat $\operatorname{Tr} 13: 474-480$

11. Prasher R, Song D, Wang JL, Phelan P (2006) Measurements of nanofluid viscosity and its implications for thermal applications. Appl Phys Lett 89:133108

12. Kulkarni DP, Das DK, Chukwu GA (2006) Temperature dependent rheological property of copper oxide nanoparticles suspension (nanofluid). J Nanosci Nanotechnol 6:1150-1154

13. He YR, Jin Y, Chen HS, Ding YL, Cang DQ, Lu HL (2007) Heat transfer and flow behaviour of aqueous suspensions of $\mathrm{TiO}_{2}$ nanoparticles (nanofluids) flowing upward through a vertical pipe. Int $\mathrm{J}$ Heat Mass Tran 50:2272-2281

14. Das SK, Choi SUS, Patel HE (2006) Heat transfer in Nanofluids - A review. Heat Transfer Eng 27:3-19 
15. Wole-Osho I, Okonkwo EC, Kavaz D, Abbasoglu S (2020) An experimental investigation into the effect of particle mixture ratio on specific heat capacity and dynamic viscosity of Al2O3-ZnO hybrid nanofluids. Powder Technol 363:699-716

16. Baghel VS, Kumar R, Roy S (2013) Heat Transfer Calculations for Decomposition of Structure I Methane Hydrates by Molecular Dynamics Simulation. J Phys Chem C 117:12172-12182

17. Rudyak V, Krasnolutskii S, Belkin A, Lezhnev E (2021) Molecular dynamics simulation of water-based nanofluids viscosity. J Therm Anal Calorim 145:2983-2990

18. Shit SP, Pal S, Ghosh NK, Sau K (2021) Thermophysical properties of graphene and hexagonal boron nitride nanofluids: A comparative study by molecular dynamics. J Mol Struct 1239:6

19. Zhou L, Ma HH (2020) A molecular dynamics study on interfacial heat transport of alkanethiol surfactant coated nanofluids-effect of chain length and stiffness. Mol Phys 118:10

20. Bao LL, Zhong CY, Jie PF, Hou Y (2019) The effect of nanoparticle size and nanoparticle aggregation on the flow characteristics of nanofluids by molecular dynamics simulation. Adv Mech Eng 11:17

21. Ding YJ, Huang BS, Shu X (2018) Modeling Shear Viscosity of Asphalt through Nonequilibrium Molecular Dynamics Simulation. Transp Res Record 2672:235-243

22. Valencia-Jaime I, Desgranges C, Delhommelle J (2019) Viscosity of a highly compressed methylated alkane via equilibrium and nonequilibrium molecular dynamics simulations. Chem Phys Lett 719:103-109

23. Chen T, Smit B, Bell AT (2009) Are pressure fluctuation-based equilibrium methods really worse than nonequilibrium methods for calculating viscosities? J Chem Phys 131:246101

24. Guo GJ, Zhang YG (2001) Equilibrium molecular dynamics calculation of the bulk viscosity of liquid water. Mol Phys 99:283-289

25. Wang X, Jing DW (2019) Determination of thermal conductivity of interfacial layer in nanofluids by equilibrium molecular dynamics simulation. Int J Heat Mass Transf 128:199-207

26. Mondello M, Grest GS (1997) Viscosity calculations of $n$-alkanes by equilibrium molecular dynamics. J Chem Phys 106:9327-9336

27. Daivis PJ, Evans DJ (1994) Comparison of constant pressure and constant volume nonequilibrium simulations of sheared model decane. J Chem Phys 100:541-547

28. Abascal JLF, Vega C (2005) A general purpose model for the condensed phases of water: TIP4P/2005. J Chem Phys 123:234505

29. Cygan RT, Liang JJ, Kalinichev AG (2004) Molecular models of hydroxide, oxyhydroxide, and clay phases and the development of a general force field. J Phys Chem B 108:1255-1266

30. Vega C, de Miguel E (2007) Surface tension of the most popular models of water by using the testarea simulation method. J Chem Phys 126:154707

31. Mao YJ, Zhang YW (2012) Thermal conductivity, shear viscosity and specific heat of rigid water models. Chem Phys Lett 542:37-41 
32. Huggins DJ (2012) Correlations in liquid water for the TIP3P-Ewald, TIP4P-2005, TIP5P-Ewald, and SWM4-NDP models. J Chem Phys 136:064518

33. Greathouse JA, Cygan RT (2006) Water structure and aqueous uranyl(VI) adsorption equilibria onto external surfaces of beidellite, montmorillonite, and pyrophyllite: Results from molecular simulations. Environ Sci Technol 40:3865-3871

34. Larentzos JP, Greathouse JA, Cygan RT (2007) An ab initio and classical molecular dynamics investigation of the structural and vibrational properties of talc and pyrophyllite. J Phys Chem C 111:12752-12759

35. Vasconcelos IF, Bunker BA, Cygan RT (2007) Molecular dynamics modeling of ion adsorption to the basal surfaces of kaolinite. J Phys Chem C 111:6753-6762

36. Plimpton S (1995) Fast Parallel Algorithms for Short-Range Molecular-Dynamics. J Comput Phys $117: 1-19$

37. Hoover WG (1985) Canonical dynamics: equilibrium phase-space distributions. Phys Rev A 31:16951697

38. Haynes WM (2016) CRC Handbook of Chemistry and Physics, 97th edn. CRC Press Inc., Boca Raton pp. 6.1-6.8

39. Perdew JP, Burke K, Ernzerhof M (1996) Generalized gradient approximation made simple. Phys Rev Lett 77:3865-3868

40. Delley B (1990) An all-electron numerical method for solving the local density functional for polyatomic molecules. J Chem Phys 92:508-517

41. Grimme S (2006) Semiempirical GGA-type density functional constructed with a long-range dispersion correction. J Comput Chem 27:1787-1799

42. Namburu PK, Kulkarni DP, Dandekar A, Das DK (2007) Experimental investigation of viscosity and specific heat of silicon dioxide nanofluids. Micro Nano Lett 2:67-71

43. Namburu PK, Kulkarni DP, Misra D, Das DK (2007) Viscosity of copper oxide nanoparticles dispersed in ethylene glycol and water mixture. Exp Therm Fluid Sci 32:397-402

44. Gonzalez MA, Abascal JLF (2010) The shear viscosity of rigid water models. J Chem Phys 132:096101

45. Harkless JAW, Stillinger DK, Stillinger FH (1996) Structures and energies of $\mathrm{SiO}_{2}$ clusters. J Phys Chem 100:1098-1103

46. Gregory JK, Clary DC, Liu K, Brown MG, Saykally RJ (1997) The water dipole moment in water clusters. Science 275:814-817

47. Bicerano J, Douglas JF, Brune DA (1999) Model for the viscosity of particle dispersions. J Macromol Sci Rev Macromol Chem Phys C 39:561-642

48. Brinkman HC (1952) The viscosity of concentrated suspensions and solutions. J Chem Phys 20:571-581 


\section{Tables}

Table 1 Calculated viscosities and density of the EMD simulations

\begin{tabular}{lllll} 
& This work & \multicolumn{3}{l}{ Experimental [38] } \\
\hline Temp & Viscosity & Density & Viscosity & Density \\
\hline $\mathrm{K}$ & $\mu_{w} / \mathrm{mPa} \bullet \mathrm{s}$ & $\mathrm{g} / \mathrm{cm}^{3}$ & $\mu_{w} / \mathrm{mPa} \bullet \mathrm{s}$ & $\mathrm{g} / \mathrm{cm}^{3}$ \\
\hline 280 & $1.357 \pm 0.032$ & 0.986 & $1.428(280.16 \mathrm{~K})$ & 1.000 \\
\hline 290 & $1.031 \pm 0.041$ & 0.978 & $1.081(290.16 \mathrm{~K})$ & 0.999 \\
\hline 300 & $0.846 \pm 0.016$ & 0.980 & $0.851(300.16 \mathrm{~K})$ & 0.997 \\
\hline 310 & $0.667 \pm 0.009$ & 0.980 & $0.692(310.16 \mathrm{~K})$ & 0.993 \\
\hline 320 & $0.538 \pm 0.029$ & 0.980 & $0.576(320.16 \mathrm{~K})$ & 0.989 \\
\hline 330 & $0.486 \pm 0.025$ & 0.976 & $0.488(330.16 \mathrm{~K})$ & 0.985 \\
\hline 340 & $0.438 \pm 0.023$ & 0.965 & $0.421(340.16 \mathrm{~K})$ & 0.979
\end{tabular}

Figures 


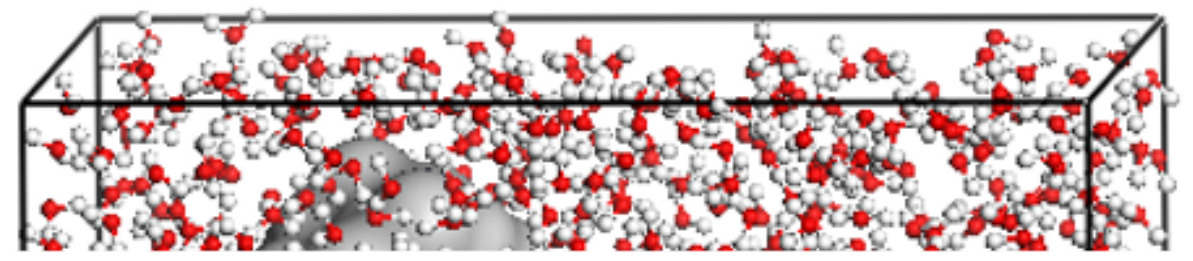

Figure 1

A snapshot of quartz particles (grey balls) in a cubic box filled with water 


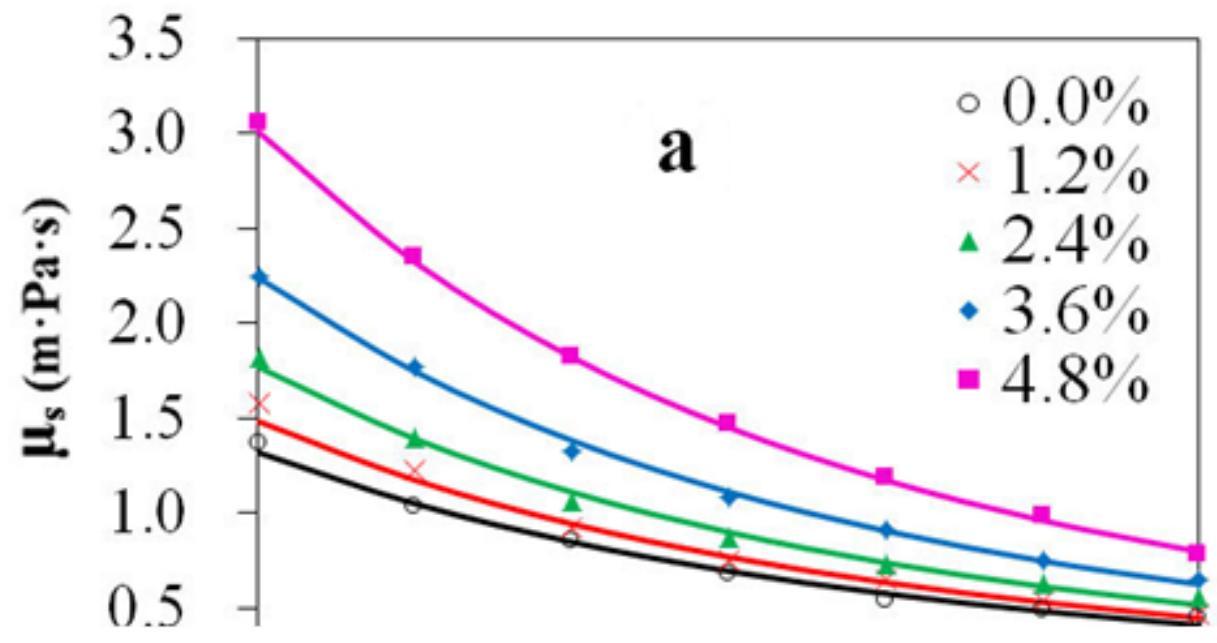

Figure 2

Computed viscosity (a) and relative viscosity (b) of quartz nanofluids at different volume concentrations and temperatures

Figure 3

Computed viscosity of quartz nanofluids with different particle diameters 


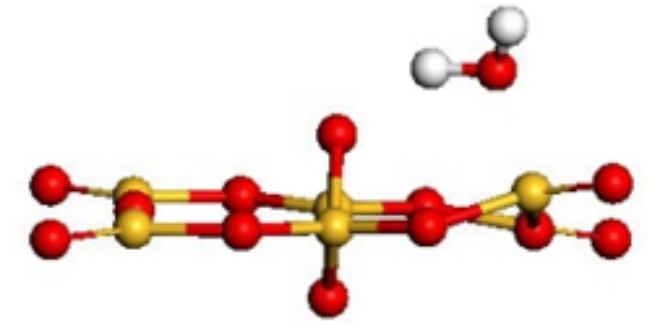

a

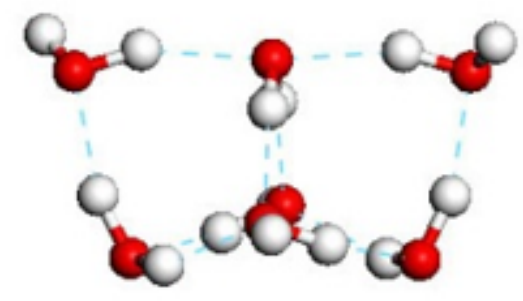

$\mathrm{b}$

م.

\section{Figure 4}

Structure models for DFT calculations. A water molecule adsorption on a $\left(\mathrm{SiO}_{2}\right)_{6}$ cluster $(\mathrm{a}),\left(\mathrm{H}_{2} \mathrm{O}\right)_{6}$ cluster (b), $\mathrm{SiO}_{2}(001)$ (c), and ice (001) 


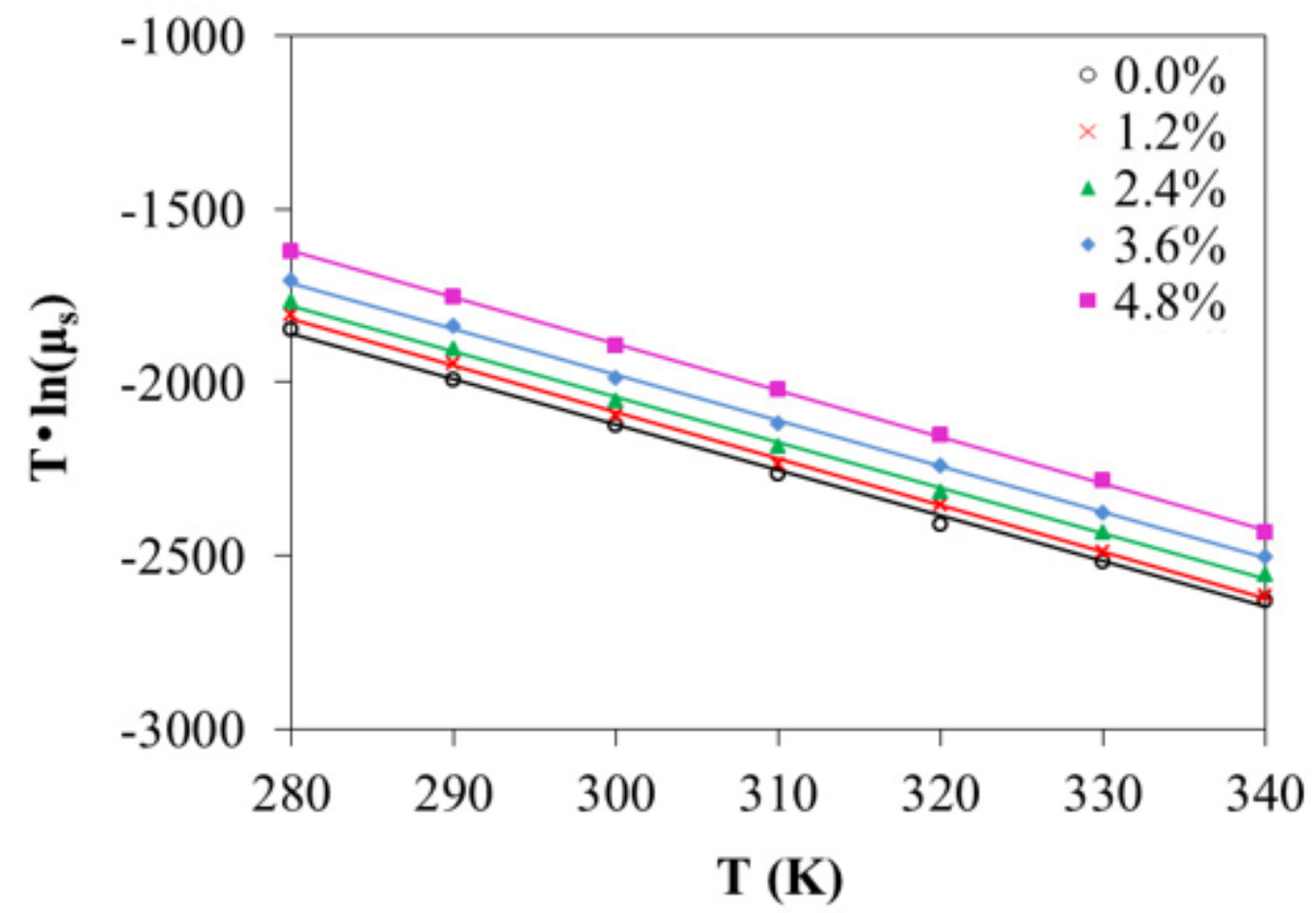

Figure 5

Correlation of $T \cdot \ln \left(\mu_{\mathrm{s}}\right)$ with temperature $(\mathrm{T})$ fitted with $\mu_{\mathrm{s}}=\mathrm{Ae}-\mathrm{B} / \mathrm{T}$

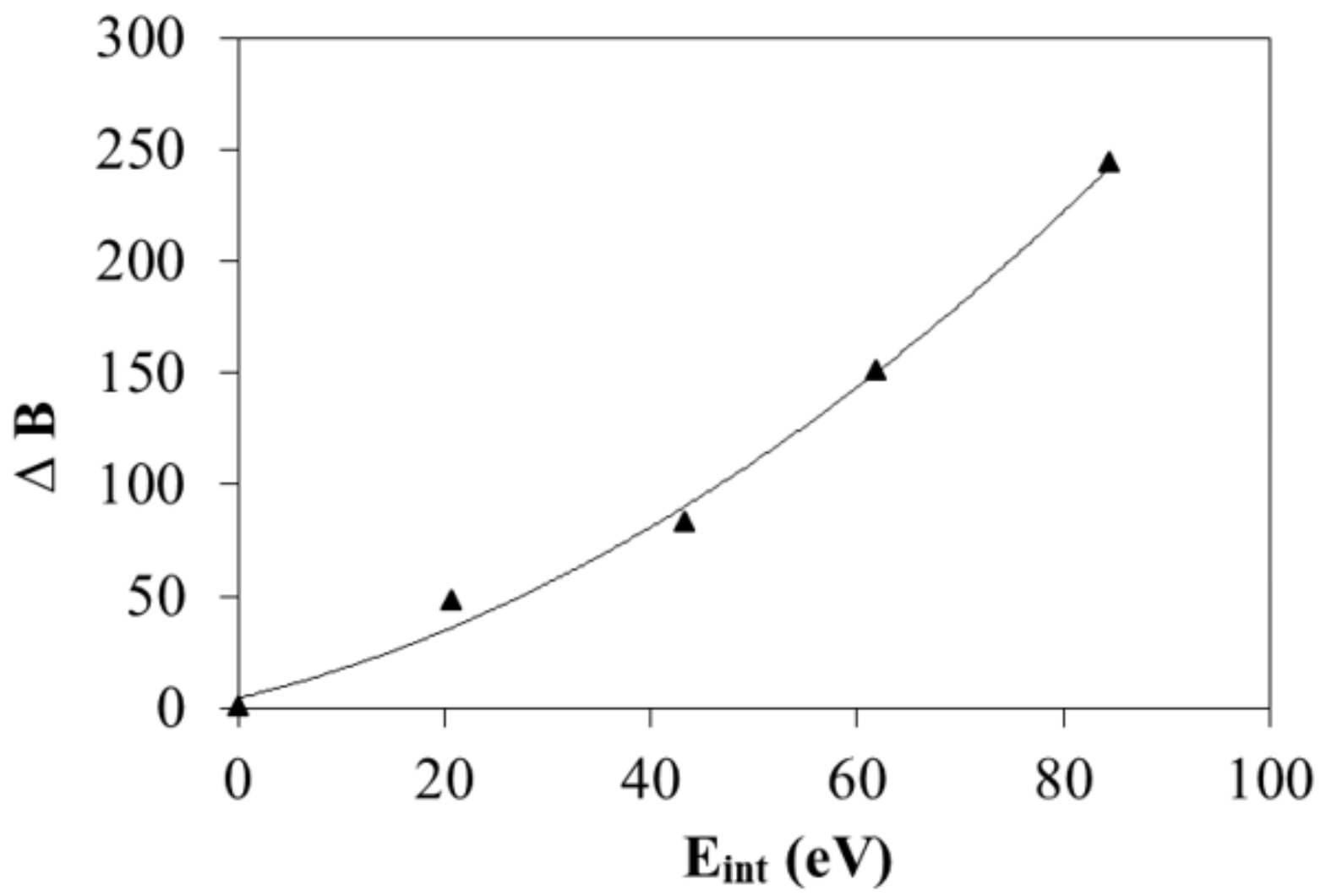


Figure 6

Correlation of $\Delta \mathrm{B}$ with $\mathrm{SiO}_{2}$-water interaction energy $\left(\mathrm{E}_{\mathrm{int}}\right)$ 